\title{
RELATIONSHIPS BETWEEN LEFT VENTRICULAR MASS, LEFT VENTRICULAR WORK AND CORONARY ARTERY SIZE IN AORTIC REGURGITATION _ Possible Mechanism of Myocardial Ischemia -
}

\author{
Kenkichi Miyahara, M.D., Masahiro Sonoda, M.D., Tadashi Kukihara, M.D** \\ Shigeru Amitani, M.D.*, Hiroshi Miyanohara, M.D**, Hideaki KaKura, M.D* \\ Kazuhiko Nakamura, M.D* and Terukatsu Arima, M.D**
}

To investigate the relationships between coronary artery size, left ventricular (LV) mass, and LV stroke work in aortic regurgitation (AR), these values were measured in 19 patients with severe AR. Twenty normal subjects and 15 patients with mitral regurgitation (MR) were used as control groups.

The coronary area index, i.e., the coronary cross-sectional area divided by body surface area (BSA), was larger in the AR group than in the control groups in all measured sites except for the peripheral left anterior descending coronary artery (LAD) and right coronary artery (RCA). However, the coronary area index divided by the LV mass was significantly smaller in AR and MR patients than in normal subjects. Furthermore, the coronary area index divided by LV stroke work was smaller in AR patients than in MR patients and normal subjects.

These results suggest that the coronary blood flow associated with the increased LV mass and stroke work caused by regurgitation was insufficient in patients with severe AR, especially in the area of the LAD. Therefore, the occurrence of myocardial ischemia in patients with severe AR may involve inadequate enlargement of the coronary artery which perfuses the $\mathrm{LV}$, in addition to factors such as decreased coronary perfusion pressure, increased coronary artery resistance and decreased coronary flow reserve.

(Jpn Circ J 1993; 57: 263-271)

$\mathbf{I}^{\mathrm{N}}$ chronic severe aortic regurgitation (AR), patients sometimes show angina pectoris despite the absence of significant coronary artery stenoses. Myocardial ischemia in patients with severe AR probably occurs due to an unbalanced blood supply and oxygen demand in the myocardium,

Key words:

Coronary blood flow

Left ventricular hypertrophy

Coronary flow reserve

Myocardial ischemia which is caused by decreased coronary blood flow secondary to reduced diastolic aortic pressure, despite the increased oxygen demand in the myocardium due to efferent left ventricular (LV) hypertrophy and increased systolic blood pressure.,2

Meanwhile, LV mass and volume of patients with chronic severe AR are greater than in patients with any other type of heart disease1. The relationship between LV mass and coronary artery diameter, or size, in

(Received April 30, 1992; accepted August 21, 1992)

Cardiovascular Division, Shinkyo Hospital, Kagoshima, ${ }^{*}$ The Second Department of Internal Medicine, Faculty of Medicine, Kagoshima University, Kagoshima

Mailing address: Kenkichi Miyahara, M.D., Cardiovascular Division, Shinkyo Hospital Usuki 3-41-1, Kagoshima 890, Japan 


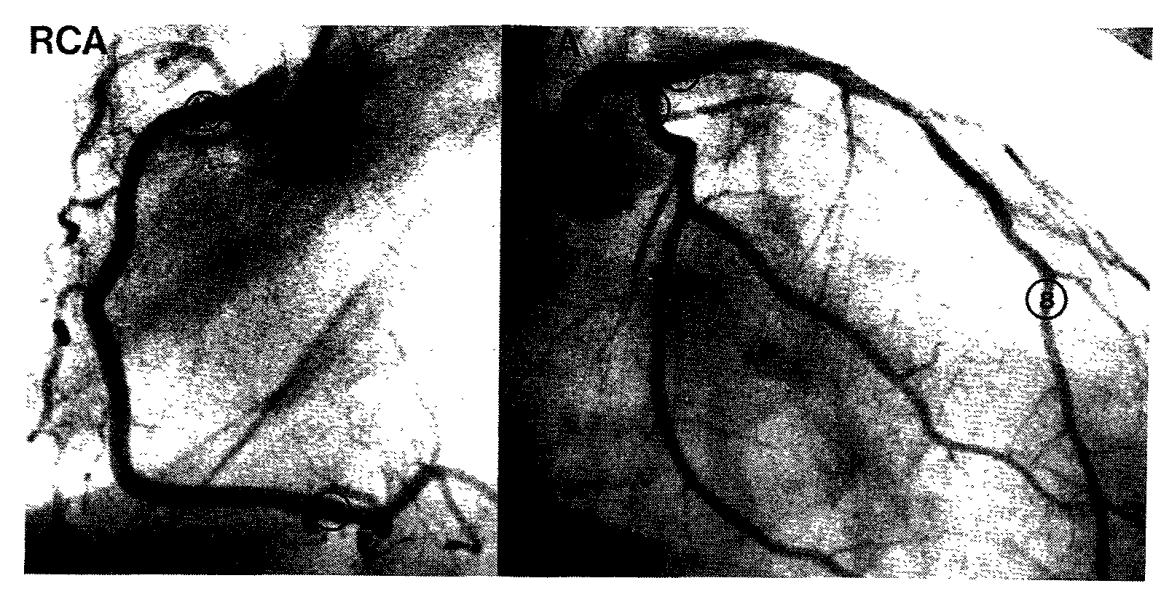

Fig.1. Coronary diameters were calculated at segments 1 and 3 of the RCA, segment 5 of the left main trunk, segments 6 and 8 of the LAD, and segment 11 of the LCX, as shown in the representative coronary arteriogram. RCA: right coronary artery, LAD: left anterior descending artery, LCx: left circumflex artery.

TABLE I CLINICAL CHARACTERISTICS AND CORONARY SIZE IN 3 GROUPS

\begin{tabular}{|c|c|c|c|}
\hline & Normal & $M R$ & $A R$ \\
\hline Number & 20 & 15 & 19 \\
\hline Age & $55.9 \pm 10.2$ & $51.4 \pm 9.6$ & $51.6 \pm 10.2$ \\
\hline $\operatorname{Sex}(M: F)$ & $10: 10$ & $9: 6$ & $17: 2$ \\
\hline$B S A\left(m^{2}\right)$ & $1.63 \pm 0.15$ & $1.59 \pm 0.18$ & $1.63 \pm 0.14$ \\
\hline$L V$ mass $(g)$ & $132.8 \pm 30.1$ & $164.9 \pm 42.6$ & $269.4 \pm 107.4^{* * *+1}$ \\
\hline$S V($ ml/beat $)$ & $64.7 \pm 15.3$ & $102.8 \pm 14.7^{* * *}$ & $112.4 \pm 27.5^{* * * m}$ \\
\hline$L V S P(m m H g)$ & $137.4 \pm 23.0$ & $131.1 \pm 18.3$ & $162.9 \pm 25.0^{* * * * m}$ \\
\hline \multicolumn{4}{|c|}{ Coronary size $\left(\mathrm{mm}^{2}\right)$} \\
\hline Seg 5 & $15.0 \pm 5.8$ & $16.9 \pm 5.6$ & $27.2 \pm 6.6^{* * *+}$ \\
\hline 6 & $10.9 \pm 2.9$ & $10.0 \pm 3.4$ & $14.9 \pm 5.1^{* *+}$ \\
\hline 8 & $3.7 \pm 1.6$ & $3.3 \pm 1.2$ & $3.6 \pm 1.2$ \\
\hline 11 & $7.5 \pm 2.9$ & $8.0 \pm 2.8$ & $11.3 \pm 4.8^{* *+}$ \\
\hline 1 & $12.0 \pm 5.1$ & $12.7 \pm 6.1$ & $21.2 \pm 9.1^{*+}$ \\
\hline 3 & $7.9 \pm 3.3$ & $7.0 \pm 2.7$ & $10.5 \pm 6.1^{*+}$ \\
\hline
\end{tabular}

*: vs normal, $+:$ vs $M R,{ }^{*}: p<0.05, * *+: p<0.01, * * *+: p<0.001$

MR: mitral regurgitation, AR: aortic regurgitation, BSA: body surface area, LV: left ventricular, SV: stroke volume, SP: systolic pressure, EDP: end-diastolic pressure

patients with AR has only been studied by a few investigators 3,4 No previous studies have examined the relationships between coronary size, LV mass, and LV stroke work. In the present study, we analyzed these relationships to determine whether or not coronary size is associated with the occurrence of myocardial ischemia in patients with chronic AR.

\section{SUBJECTS AND METHODS}

Nineteen patients with pure $A R$, who were diagnosed by aortography as grade 3 or 4 according to the Sellers classification, were selected as subjects (AR group). Fifteen patients with pure mitral regurgitation (MR) (MR group) of grade 3 or 4 according to the Sellers classification, and 20 normal cases (Normal group), who had been examined for chest pain, but had no organic heart diseases, were adopted as control groups. All of the cases showed normal sinus rhythm and no obvious coronary lesions. Clinical characteristics of the three groups are shown in Table I. 

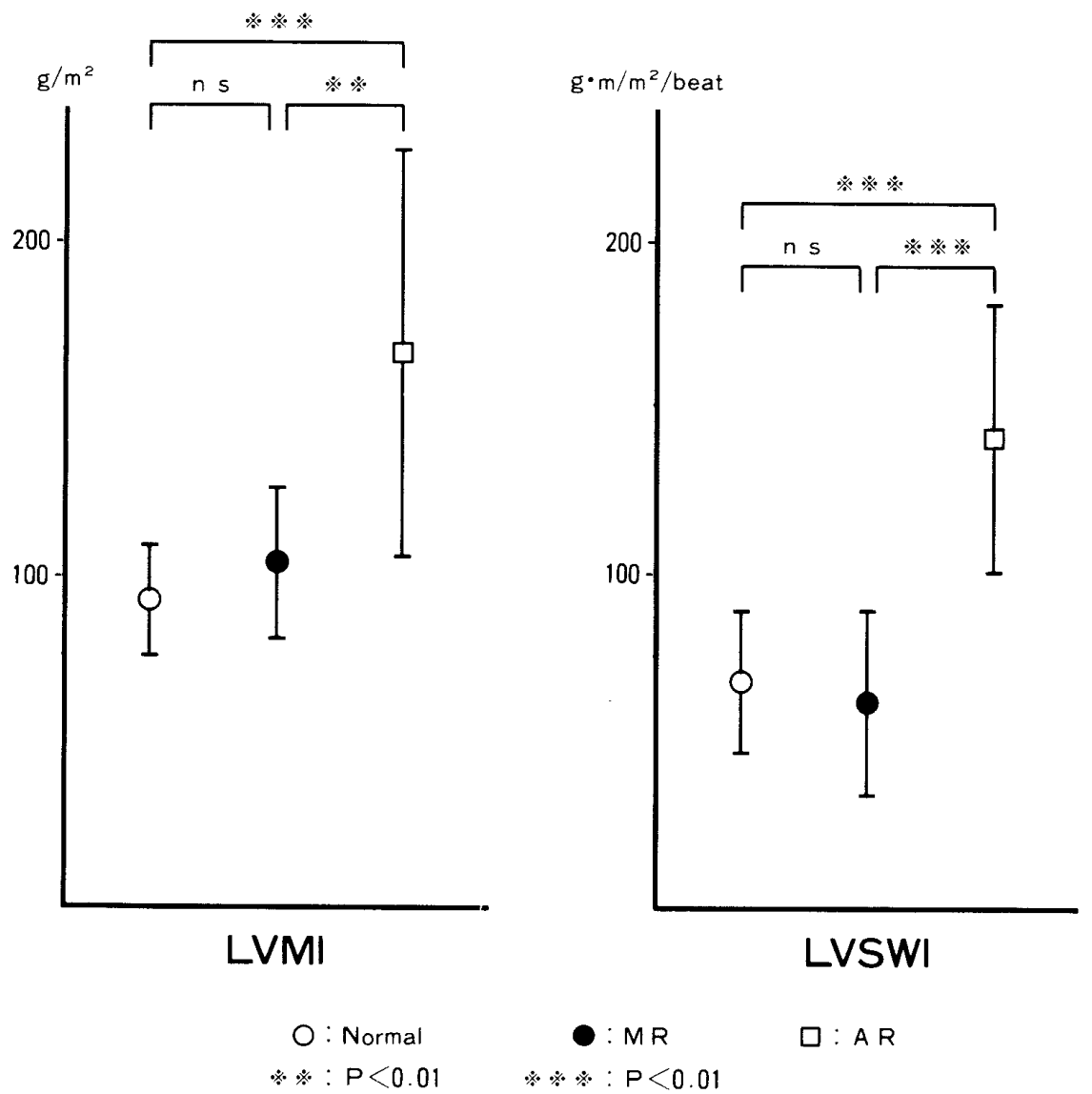

Fig.2. Comparisons of LVMI and LVSWI between the three groups. MR: mitral regurgitation, AR: aortic regurgitation, LVMI: left ventricular mass index, LVSWI: left ventricular stroke work index.

Cardiac catheterization and angiocardiography were performed by a femoral approach. The left ventricular and aortic pressures were measured by the fluid-filled method using a $6 \mathrm{~F}$ pig-tail catheter. A pressure transducer (Gould, P-3) was connected to a recorder (San-ei Polygraph 360 System) with a paper speed of $50 \mathrm{~mm} / \mathrm{sec}$. Coronary angiography was performed from at least three projections for the right coronary artery (RCA), and from three or four projections for the left coronary artery using a $6 \mathrm{~F}$ Judkins catheter. Left ventriculography and aortography were performed using a $6 \mathrm{~F}$ pigtail catheter, and a contrast medium of 0.8 to $1.0 \mathrm{ml} / \mathrm{kg}$ was used at an injection rate of 8 to $10 \mathrm{ml} / \mathrm{sec}$. Left ventriculograms were obtained from a right anterior oblique angle of $30^{\circ}$, and aortograms from a left anterior oblique angle of $60^{\circ}$. In the normal group, LV stroke volume was obtained by the thermodilution method immediately before left ventriculography. Due to the regurgitant flow in the MR and AR groups, the stroke volume was obtained from a left ventriculogram by the single plane method according to Kennedy et al6. The LV stroke work index (LVSWI) was calculated using the following equations.

$$
\begin{aligned}
& \mathrm{SI}=\mathrm{SV} / \mathrm{BSA}\left(\mathrm{ml} / \mathrm{m}^{2} / \text { beat }\right) \\
& \mathrm{LVSWI}=\mathrm{SI} \times(\mathrm{LVSP}-\mathrm{LVEDP}) \times \\
& 0.0136\left(\mathrm{~g} \cdot \mathrm{m} / \mathrm{m}^{2} / \text { beat }\right)
\end{aligned}
$$

(SI: stroke index, BSA: body surface area, LVSP: left ventricular systolic pressure, LVEDP: left ventricular end-diastolic pressure)

The LV mass was measured by the method of Rackley et al? The magnification rate was corrected by calculation based on scales, which were placed on the front and back surfaces of the subjects. The LV mass was divided by the BSA of each subject to obtain the LV mass index. The coronary artery diameter was measured using the 


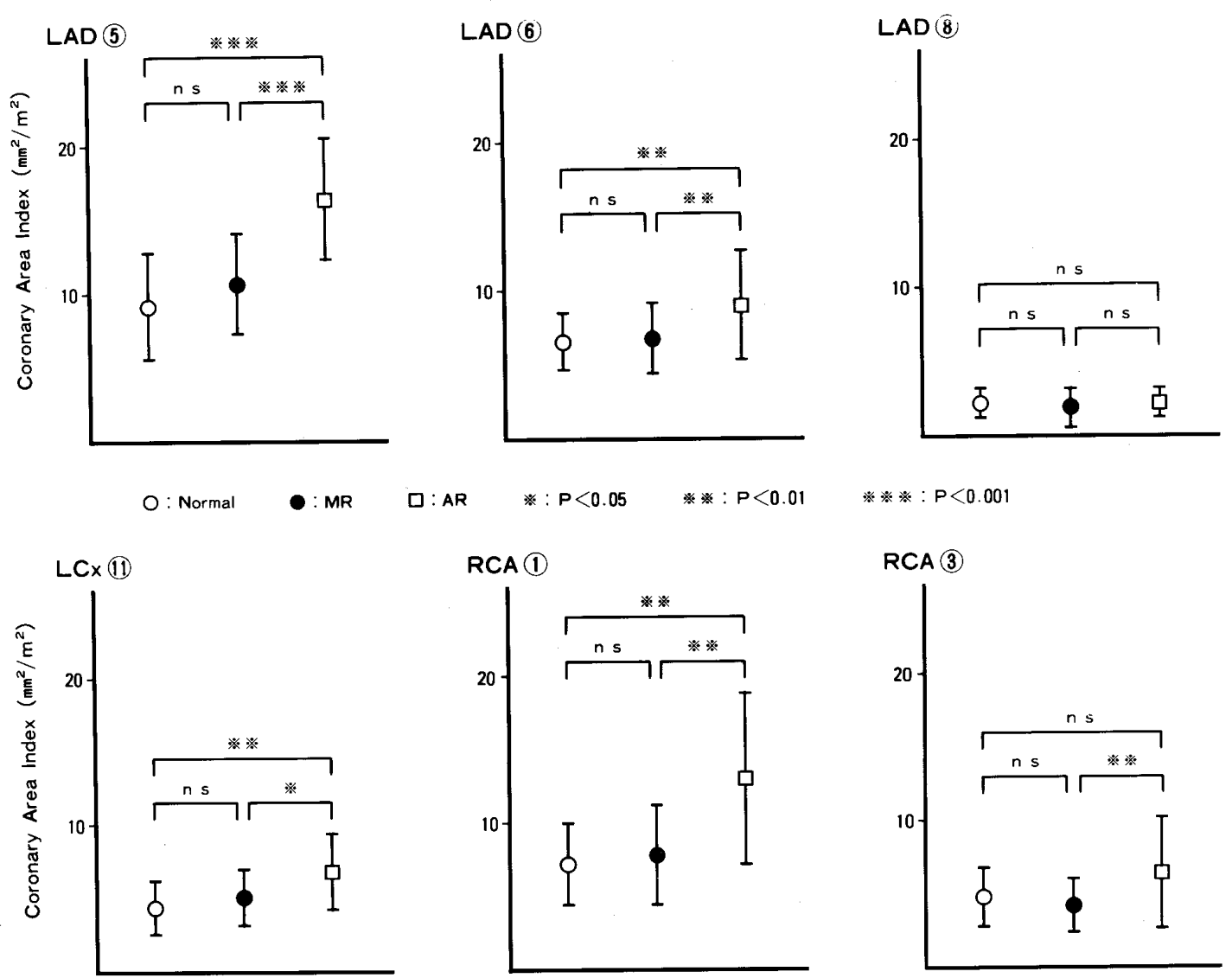

Fig.3. Coronary area indexes in each segment and comparisons between the 3 groups. MR: mitral regurgitation, AR: aortic regurgitation.

end-diastolic angiograms from a projection which enabled the target site to be clearly separated, and the magnification rate was corrected by the diameter of a $6 \mathrm{~F}$ Judkins catheter. The six sites for measurement of the coronary artery diameter were targeted according to the AHA classification: proximal of segment 1 and distal of segment 3 of the RCA, segment 5 of the left main trunk, proximal of segment 6 and proximal of segment 8 of the left anterior descending artery (LAD), and proximal of segment 11 of the left circumflex artery (LCx) (Fig. 1). The peripheral branch of the LCx was not used as a target site because it was difficult to compare due to its many variations. The coronary artery diameter was measured by two observers, and the mean value was used. The cross-sectional area of the coronary artery was calculated from the coronary diameter and expressed as the coronary size. Quantitative evaluation of coronary angiogram, LV mass and volume were performed with a semiautomatic computer system based on a $35 \mathrm{~mm}$ cine-projector (ELK, CAP-
35B).

All data were expressed as the mean \pm standard deviation. Statistical analyses were performed by the non-paired t test, and correlation was analyzed by the least squares linear regression equation. $\mathrm{P}$ values less than $5 \%$ were considered statistically significant.

\section{RESULTS}

The results observed for all of the groups are shown in Table I. No significant differences were observed between the age or BSA in the 3 groups. The LV mass and systolic pressure were significantly higher in the AR group than in the other 2 groups. The LV stroke volume was significantly larger in the MR group than in normal group, and significantly larger in the AR group than in the MR group. LVEDP was higher in the MR and AR groups, but this difference was not statistically significant. The coronary size was apparently larger in the AR group, except for distal of the LAD, than in the 


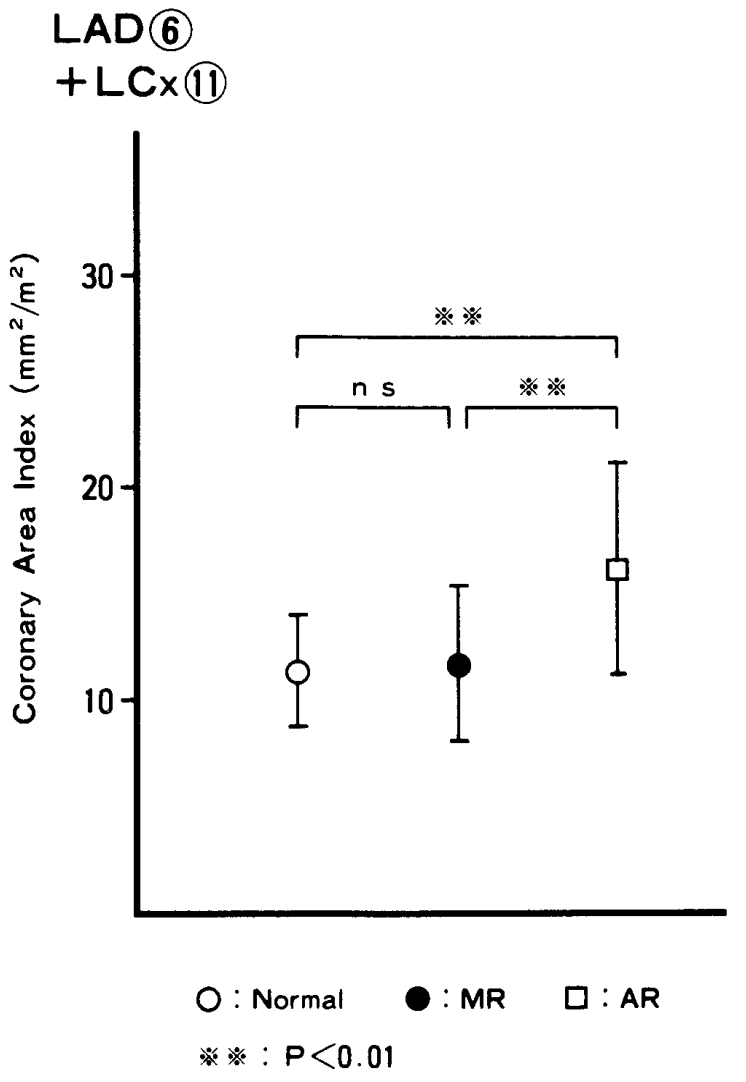

Fig.4. Comparison of coronary area indexes (LAD(6) + LCx(11) between the 3 groups. MR: mitral regurgitation, AR: aortic regurgitation.

normal and MR groups (Table I). The LV mass index and LVSWI were significantly larger in the AR group than in the other 2 groups (Fig. 2). The coronary area index, obtained by dividing the coronary size by BSA, was significantly larger in the AR group than in the normal group in all sites, except for in the peripheral LAD and RCA. It was also significantly larger in the AR group than in the MR group, except for in the main trunk of the left coronary artery. However, no significant difference was observed between the normal and the MR groups in any of the sites (Fig. 3). Furthermore, the coronary area index of the proximal LAD $+\mathrm{LCx}$, which primarily perfuses the left ventricle, was significantly larger in the AR group than in the normal and MR groups, although no significant differences were observed between the normal and MR groups (Fig. 4).

In order to investigate the coronary size per LV mass, the coronary area index divided by the LV mass index was compared at each coronary site. As shown in Fig. 5, no significant difference was recognized between the normal group and the AR group in the RCA and $\mathrm{LCx}$, which primarily perfuses the right ventricle, and the inferoposterior wall of the left ventricle. Significant differences were also not observed between the normal group and the MR group in all sites, except for in the peripheral RCA. However, the coronary area index divided by the LV mass index in the LAD was obviously lower in the AR and MR groups than in the normal group. Moreover, the coronary artery size of proximal LAD $+\mathrm{LCx}$ per LV mass was significantly smaller in the $A R$ and MR groups than in the normal group (Fig. 6). The coronary size per LVSWI was significantly smaller in the AR group than in the normal group in the LAD and the peripheral RCA, and tended to be smaller than the normal group in other sites. Meanwhile, the coronary size per LVSWI was significantly larger in the MR group than in the normal group in the left main trunk, and tended to be larger in other sites, Furthermore, it was significantly larger in the MR group than in the AR group in all sites (Fig. 7). In addition, dividing the coronary artery size of proximal $\mathrm{LAD}+\mathrm{LCx}$ by $\mathrm{LV}$ stroke work revealed a significant lower value in the AR group than in the other two groups (Fig. 6).

\section{DISCUSSION}

Myocardial ischemia is produced by a greater oxygen demand than the current oxygen supply; i.e., relatively decreased coronary blood flow. Coronary blood flow is influenced by various factors, including the coronary diameter, coronary perfusion pressure, inner-diameter of the resistant vessels, intramyocardial pressure and blood viscosity, and so on? Rouleau et $\mathrm{al}^{9}$ reported that coronary blood flow did not change due to autoregulation of the resistant vessels, even though the coronary perfusion pressure changed dramatically. However, these resistant vessels lose the ability to autoregulate, and the coronary flow reserve is reduced, in patients with hypertrophic heart, such as hypertrophic cardiomyopathy or aortic stenosis. It is believed that these phenomena play an important role in the occurrence of 


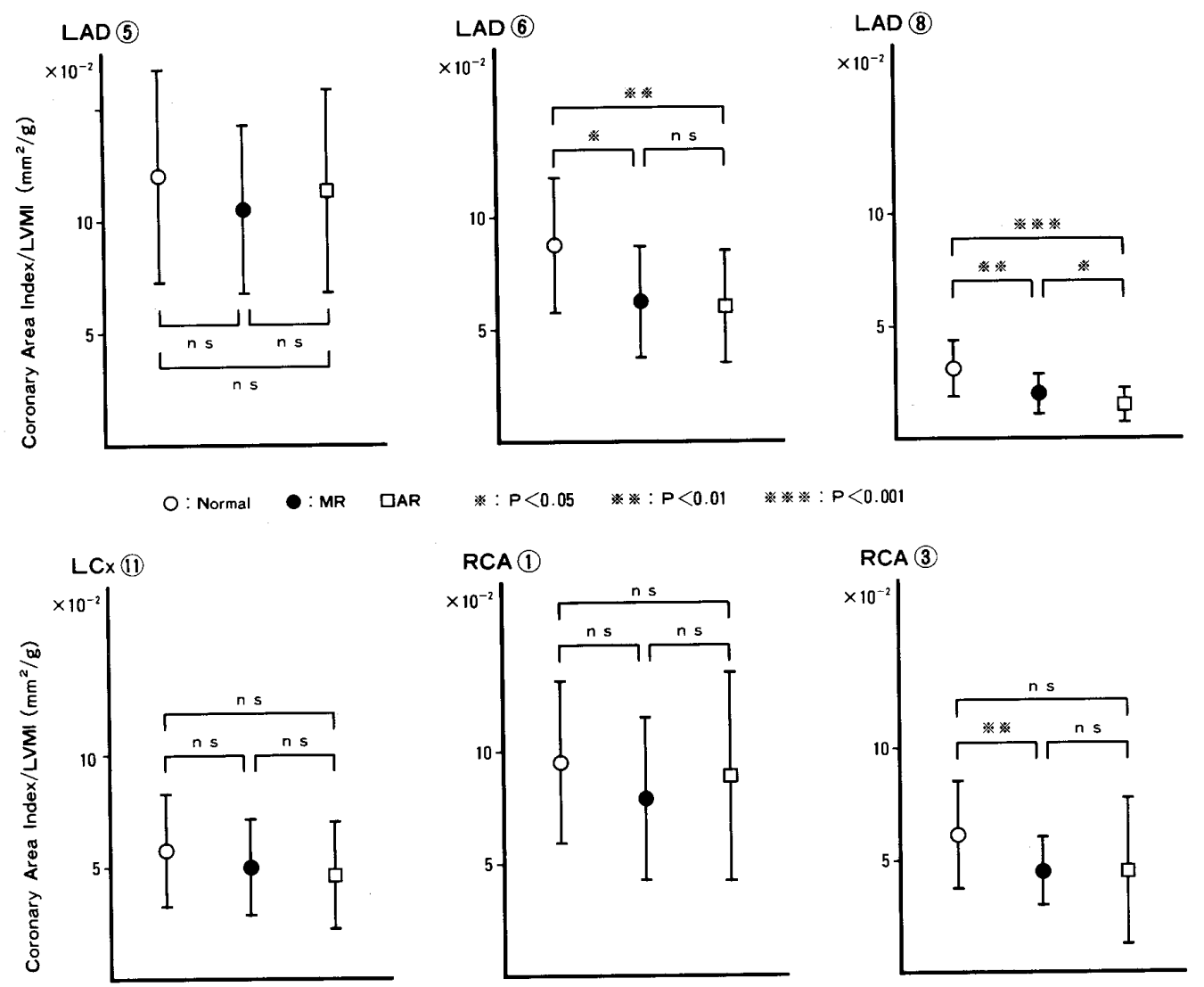

Fig.5. Coronary area index per LVMI in each segment and comparisons between the three groups. MR: mitral regurgitation, AR: aortic regurgitation, LVMI: left ventricular mass index.

myocardial ischemia ${ }^{10-12}$ Kimball et al ${ }^{13}$ studied the coronary size in patients with aortic stenosis or hypertrophic cardiomyopathy. They reported that although the coronary size was increased in hypertrophic hearts, the degree of increase in the coronary size was "inadequate" compared with the degree of increase in the LV mass, which may cause anginal pain in patients with hypertrophic cardiomyopathy or aortic stenosis. Reduced coronary flow reserve and perfusion pressure may also be strongly associated with the occurrence of myocardial ischemia in patients with AR. We cannot neglect the possibility that coronary size is also involved in the occurrence of myocardial ischemia, as stated by Kimball et al1 ${ }^{13}$

Since the flow volume in a lumen is theoretically proportional to its crosssectional area $(\mathrm{Q}=\mathrm{vA}$, where $\mathrm{Q}$ : flow volume, v: flow velocity, and A: cross-sectional area of the lumen), the coronary blood flow should be increased in proportion to the squared diameter of the coronary artery. Therefore, besides determining various aspects of coronary blood flow, such as coronary perfusion pressure, the resistance of coronary vessels, intramyocardial pressure and so forth $1,2,14,15$ the coronary artery size is also an important factor in the magnitude of the coronary blood flow itself. On the other hand, the occurrence of myocardial ischemia is caused by the relative decrease of coronary blood flow per myocardial mass, or the relative increase of myocardial oxygen demand versus the coronary blood flow. Based on this viewpoint, we believed that relationships between the coronary artery size, myocardial weight and LV work should be analyzed to clarify the mechanism which may cause myocardial ischemia in AR without apparent coronary artery stenosis.

In the present study, the coronary size was larger in the AR group than in the normal and MR groups in all of the measured coronary sites, except for in the peripheral LAD and RCA. However, the coronary size of 


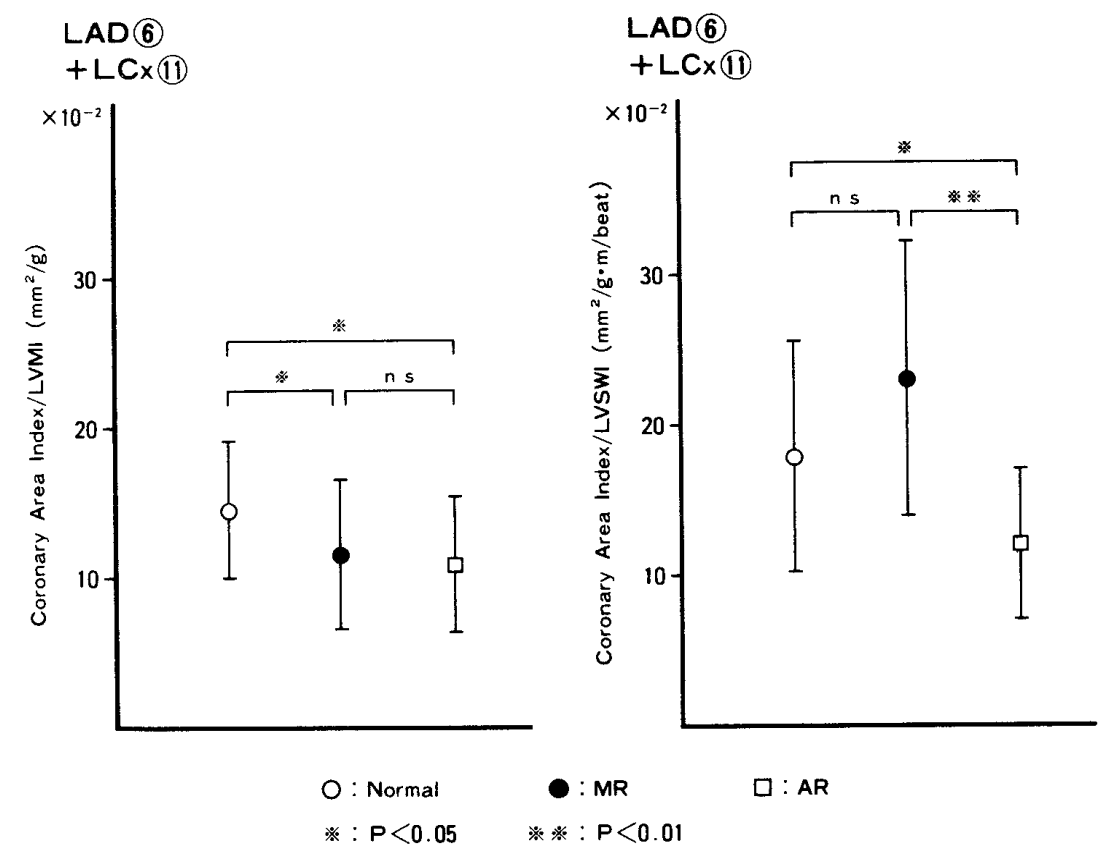

Fig.6. Comparisons of coronary area index (LAD(6) + LCx(11)) per LVMI and LVSWI between the 3 groups. MR: mitral regurgitation, AR: aortic regurgitation, LVMI: left ventricular mass index, LVSWI: left ventricular stroke work index.

$\mathrm{LAD}+\mathrm{LCx}$ per $\mathrm{LV}$ mass was significantly smaller in the AR group than in the normal group. The coronary size of $\mathrm{LAD}+\mathrm{LCx}$ per LVSWI was also significantly smaller in the AR group than in both the normal and MR groups. In addition, for each in the coronary artery, the coronary size per LV mass and LV stroke work was significantly smaller in the peripheral LAD in the AR group than in the other two groups. These results suggest that, in patients with severe AR, sufficient coronary blood flow, corresponding to an increased LV mass and stroke work caused by $A R$, is not supplied to the left ventricle, especially in the area perfused by the LAD.

Lewis and Gotsman ${ }^{3}$ studied the relationship between the coronary cross-sectional area and LV mass in patients with various heart diseases, including AR. They reported that there was an almost linear relationship between the left ventricular mass and coronary size. However, in their report, the relationship between the coronary size and LV work was not explained. Furthermore, the stroke indexes in patients with AR were similar to those in normal subjects. Therefore, we believe that many relatively mild AR cases may have been included in their study. On the other hand, Cannon et al ${ }^{12}$ re- ported that the coronary blood flow at rest was significantly increased in patients with hypertrophic cardiomyopathy, as compared with control groups. Negishi et $\mathrm{al}^{14}$ also stated that the diastolic coronary blood flow at rest was increased in patients with hypertrophic cardiomyopathy. In addition, Paulsen et $\mathrm{al}^{16}$ and Roberts and Roberts ${ }^{17}$ showed that the left coronary artery diameter was significantly larger in hypertrophic hearts than in normal hearts. However, whether or not the increased coronary size and coronary blood flow were adequate for the increased LV mass, was not investigated in these reports. Recently, Villari et $\mathrm{al}^{4}$ reported that a correlation existed between coronary artery dimensions and the left ventricular mass before and after valve replacement in patients with aortic valve disease. They concluded that the proximal coronary artery size was increased before surgery, but its increase was not sufficient to maintain coronary size per $100 \mathrm{~g}$ of LV mass. These reports and our present study suggest that although coronary size and coronary blood flow are increased in patients with severe AR, the magnitudes of these increases are inadequate compared to the increase in the left ventricular mass and stroke work, resulting in a relative decrease of coronary blood 

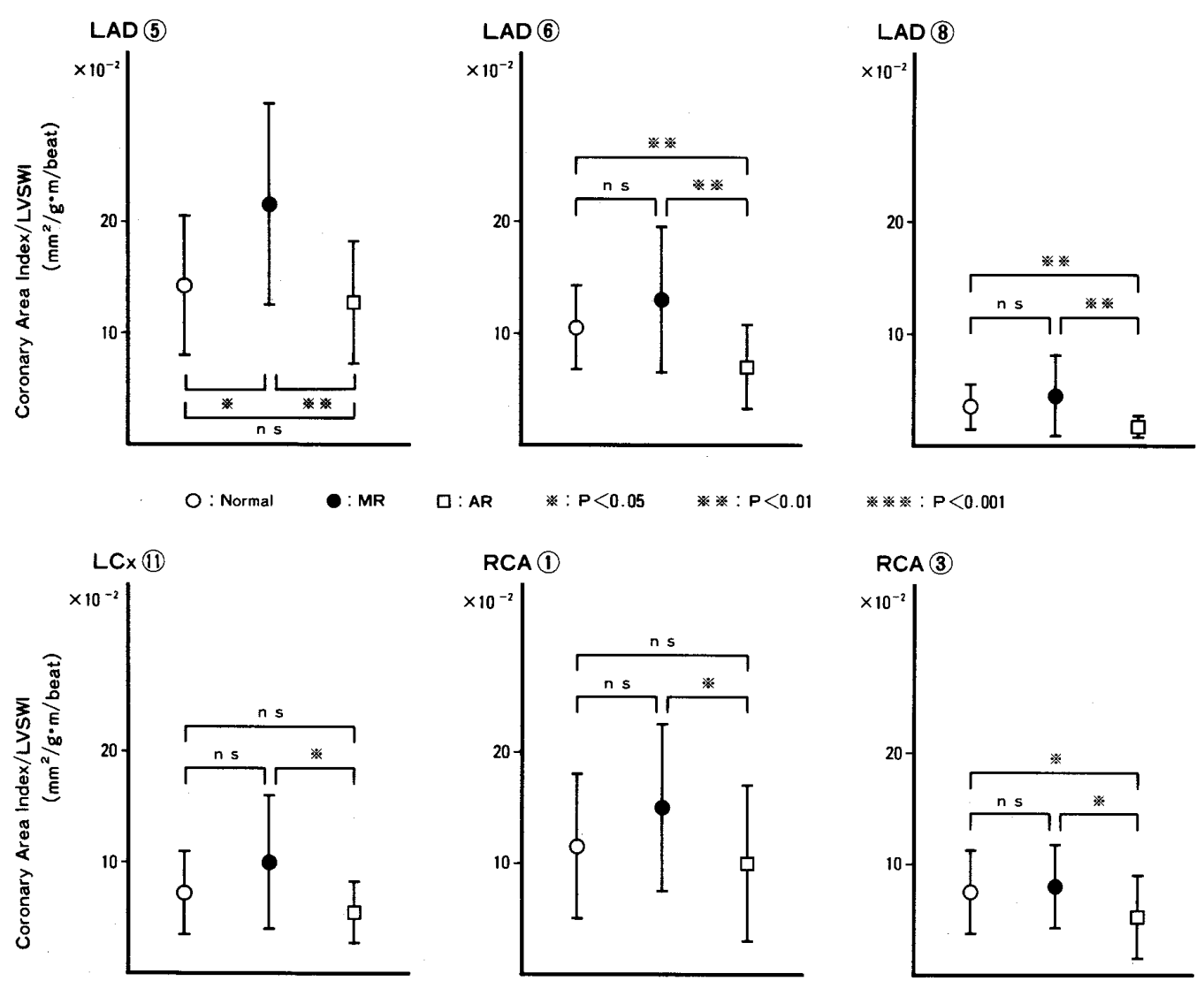

Fig.7. Coronary area index per LVSWI in each segment and comparisons between the 3 groups. MR: mitral regurgitation, AR: aortic regurgitation, LVSWI: left ventricular stroke work index.

flow.

If the diameter of the outflow tract is smaller than the inflow, the resistance in the outflow tract, would theoretically increase. In this study, it was revealed that the proximal coronary size was significantly larger in the AR group than in the other two groups, in spite of no difference in the peripheral LAD. Therefore, the resistance of the coronary artery may have been relatively increased in the peripheral LAD in patients with AR. Thus, the occurrence of myocardial ischemia in patients with severe AR is probably associated with "inadequate enlargement" of the coronary artery which perfuses the left ventricle, in addition to previously reported factors, such as decreased coronary perfusion pressure, increased coronary resistance, decreased coronary flow reserve, and so on! $1,2,14,15$

Regarding a mechanism for the increase of proximal coronary artery size in AR patients, one report has suggested that it is an adaptive response to maintain the coronary flow velocity, or the result of some humoral factors, such as endothelium-derived vasoactive factors4 However, we were unable to identify such a mechanism. In addition, since only coronary size was measured, rather than the actual coronary blood flow, it may be impossible to clarify all of the mechanisms for the occurrence of myocardial ischemia in patients with AR. Furthermore, since a comparison between patients with and without anginal pain, or a comparison between patients with severe and mild AR was not conducted, it is difficult to say that a full investigation was performed. Therefore, further investigation involving a direct measurement of the coronary blood flow in more patients will be required.

\section{Acknowledgments}

We gratefully acknowledge Prof. Yasuo Morishita (The Second Department of Surgery, Gunma University) for his helpful comments. 


\section{REFERENCES}

1. BRAUNWALD E: Valvular heart disease. In: BRAUNHALD E, ed. Heart disease. 4th ed. Philadelphia, WB Saunders Co, 1992: 1043-1053

2. FALSETTI HL, CARROLL RJ, CRAMER JA: Total and regional myocardial blood flow in aortic regurgitation. Am Heart $J$ 1979; 97: 485-493

3. LEWIS BS, GOTSMAN MS: Relation between coronary artery size and left ventricular wall mass. Br Heart J 1973; 35: 1150-1153

4. VILLARI B, HESS OM, MEIER C, PUCILLO A, GAGLONE A, TURINA $M$, KRAYENBUEHL HP: Regression of coronary artery dimensions after successful aortic valve replacement. Circulation 1992; 85: 972-978

5. SELLERS RD, LEVY MJ, AMPLATZ K, LELLEHEI CW: Retrograde cardioangiography in acquired cardiac disease: technique, indications and interpretation of 700 cases. Am J Cardiol 1964; 14: $437-447$

6. KENNEDY JW, TRENHOLME SE, KASSER IS: Left ventricular volume and mass from single plane cineangiocardiogram. A comparison of anteroposterior and right anterior oblique methods. $\mathrm{Am}$ Heart $J$ 1970; 80: $343-352$

7. RACKLEY CE, DODGE HT, COBEL YD JR, HAY RE: A method for determining left ventricular mass in man. Circulation 1964; 29: 666-671

8. SCHLANT RC, SONNENBLICK EH: Normal physiology of the cardiovascular system, in HURST JW ed. The Heart. 6th ed. New York, McGraw-Hill Book Co. 1986: 60-63

9. ROULEAU J, BOERBOOM LE, SURJADHANA A, HOFFMAN JIE: The role of auto-regulation and tissue diastolic pressures in the transmural distribution of left ventricular blood flow in anesthetized dogs. Circ Res 1979; 45: 804-815

10 OPHERK D, MALL G, ZEBE H, SCHWARZ F, WEIHE E, MANTHEY J, KUBLER W: Reduction of coronary reserve: a mechanism for angina pectoris in patients with arterial hypertension and normal arteries. Circulation 1984; 69: $1-7$

11 MARCUS $M$, DOTY DB, HIRATZKA LF, WRIGHT CB, EASTHAM CL: Decreased coronary reserve: a mechanism for angina pectoris in patients with aortic stenosis and normal coronary arteries. N Engl J Med 1982; 307: 1362-1366

12 CANNON RO, ROSING DR, MARON BJ, LEON MB, BONOW RO, WATSON RM, EPSTEIN S: Myocardial ischemia in patients with hypertrophic cardiomyopathy: contribution of inadequate vasodilator reserve and elevated left ventricular filling pressures. Circulation 1985; 71: $234-243$

13 KIMBALL BP, LIPRETI V, BUI S, WIGLE ED: Comparison of proximal left anterior descending and circumflex coronary artery dimensions in aortic valve stenosis and hypertrophic cardiomyopathy. Am J Cardiol 1990; 65: 767-771

14 NEGISHI K, HANDA S, ASAKURA Y, IWANAGA S, ISHIKAWA S, WAINAI Y, ABE S, TANI M: Coronary flow characteristics in hypertrophic cardiomyopathy. A study with Doppler catheter. Kokyu To Junkan 1991; 39: 1021-1027 (in Japanese)

15 NITENBERG A, FOULT JM, ANTONY I, BLANCHET F, RAHALI M: Coronary flow and resistance reserve in patients with chronic aortic regurgitation, angina pectoris, and normal coronary arteries. $J$ Am Coll Cardiol 1988; 11: $478-486$

16 PAULSEN S, VETNER M, HAGERUP LM: Relationship between heart weight and the cross sectional area of the coronary ostia. APMIS 1975; 83: $429-432$

17 ROBERTS CS, ROBERTS WC: Cross-sectional area of the proximal portions of the three major epicardial coronary arteries in 98 necropsy patients with different coronary events. Relationship to heart weight, age and sex. Circulation 1980; 62: 953-959 